http://dx.doi.org/10.5007/2175-7984.2012v11n20p135

\title{
Biotecnología agrícola y "sojización" en la Argentina: controversia pública, construcción de consenso y ampliación del marco regulatorio
}

\section{Resumen}

La incorporación de transgénicos en la agricultura argentina con una orientación agroexportadora, desde mediados de la década del noventa, fue liderada por la soja tolerante a herbicida. En los inicios, la resistencia pública a esa incorporación no tuvo el peso como para modificar de manera sustancial los planes de sus promotores, entre los que se cuenta un marco regulatorio que se cerró tempranamente en torno a los riesgos biofísicos. Sin embargo, hemos comprobado que se han ido incorporando normativas complementarias al sistema regulatorio formal. Las mismas retoman algunas críticas locales y se constituyen en una extensión informal de dicho sistema, que atiende cuestiones locales de impacto ambiental, social y de salud. Previamente, se logró también que se pusiera en debate la introducción de los transgénicos; y que se alcanzara un cierto consenso, entre actores pro y anti-transgénicos, con respecto a ciertos impactos negativos sociales y ambientales de esa introducción. Palabras clave: transgénicos, soja tolerante a herbicida, resistencia a la tecnología, marco regulatorio, Argentina.

1 Centro de Estudios de Historia de la Ciencia José Babini, Escuela de Humanidades, Universidad Nacional de San Martín (UNSAM). 
Biotecnología agrícola y "sojización" en la Argentina: controversia pública...

Ana María Vara • Agustín Piaz • Florencia Arancibia

\section{Introducción}

En el campo de la biotecnología agrícola, puede observarse un Eintento activo y de gran alcance para construir un régimen mundial de regulación, que se reproduce a nivel nacional en cada país, el que permite a un reducido número de empresas transnacionales de biotecnología agrícola, con sedes en Estados Unidos y Europa, operar en múltiples mercados, en particular facilitando la producción, comercio y de cultivos y alimentos genéticamente modificados, también llamados transgénicos (VAN ZWANENBERG, en prensa).

La incorporación de transgénicos en la agricultura argentina con una orientación agroexportadora, desde mediados de la década del noventa, fue liderada por la soja tolerante a herbicida. Si bien entusiasta y veloz, esta incorporación conoció la resistencia de ciertos sectores sociales ya desde los inicios. Sin embargo, durante los primeros años de su incorporación al mercado argentino, esa resistencia no tuvo el peso suficiente como para modificar de manera sustancial los planes de los promotores de esta tecnología; fundamentalmente, las empresas transnacionales de biotecnología agrícola y sus aliados locales (productores, empresas semilleras y de agroquímicos, áreas del Estado), que vieron en esta tecnología una oportunidad para mejorar la competitividad de la producción agrícola destinada a la exportación (VARA, 2005: cap. 1). La falta de impacto de esta resistencia pudo deberse a una serie de factores específicos de la Argentina, entre ellos, la baja conciencia pública de la rápida incorporación de transgénicos; la conformación de un frente promotor de esta tecnología que incluyó importantes actores locales (oficinas de gobierno, productores, empresas semilleros, expertos, medios de comunicación dominantes); la orientación de los grupos de consumidores hacia otras cuestiones; los beneficios económicos tanto para los productores locales (incluyendo los medianos y pequeños) como para las arcas estatales; el hecho de que la soja transgénica resultara una solución viable y ambientalmente sostenible para problemas agronómicos de la Pampa Húmeda, la zona de mayor desarrollo agropecuario históricamente; la historia 
y alta valoración pública de la Argentina como país agro-exportador (TRIGO y CAO, 2003; TRIGO et al., 2002; VARA, 2005: caps. 1 y 5; PELLEGRINI, 2010).

Para este trabajo, hemos entrevistado a representantes de la industria o cercanos a ella; del sistema regulatorio o cercanos al mismo; de la sociedad civil y asesores científicos de este sector; y de los poderes del Estado (un funcionario de la Cancillería y un diputado), con el objetivo de contribuir a comprender los procesos de rediseño de la regulación de la biotecnología agrícola en el contexto argentino. Como en muchos países (VAN ZWANENBERG, en prensa), en la Argentina existe un campo regulatorio formal, dominado por la Comisión Nacional Asesora de Biotecnología Agropecuaria (CONABIA) y la Dirección de Biotecnología del actual Ministerio de Agricultura, Ganadería y Pesca, basado en los principios de "armonización", en torno a los convenios regulatorios internacionales sobre riesgos biofísicos (seguridad de la biotecnología). Hay también un elemento propio de la Argentina: la revisión de la Dirección Nacional de Mercados Agroalimentarios, dedicada a analizar si el desarrollo en cuestión pudiera afectar las exportaciones. Este campo regulatorio, si bien fue sometido a sucesivas oleadas de críticas y protestas en la Argentina, ha permanecido casi sin variantes en su fundamentación e implementación, desde un punto de vista formal.

Sin embargo, como resultado de nuestra investigación surge que se han ido incorporando normativas más o menos generales que pueden considerarse complementarias al sistema regulatorio formal, en la medida en que retoman algunas de las críticas más importantes suscitadas en el contexto local y se constituyen en un extensión informal de dicho sistema, que atiende cuestiones locales y, por lo tanto, peculiares, de impacto ambiental, social y de salud. Hemos detectado tres líneas, a las que nos referiremos siguiendo el orden de aparición: la primera, referida al uso de la soja como alimento, en particular para niños y poblaciones vulnerables; la segunda, destinada a contener el proceso de deforestación inducido por la agriculturización marcada por la intensificación de 
Biotecnología agrícola y "sojización" en la Argentina: controversia pública...

Ana María Vara • Agustín Piaz • Florencia Arancibia

la producción de la soja transgénica; la tercera, orientada a regular el uso de ciertos agroquímicos. También detectamos indicios de una cuarta, referida a la propiedad de la tierra, a la que nos referiremos muy brevemente; además de otros aspectos que están en discusión.

Previo a estos resultados, se verificaron otros dos: que se pusiera en debate la introducción de los transgénicos en la Argentina dentro de un proceso de agriculturización orientado a la exportación de commodities; y que, como consecuencia parcial de ese debate, se alcanzara un cierto consenso, entre actores pro y anti-transgénicos incluyendo a representantes del propio sistema regulatorio, con respecto a ciertos significativos impactos negativos sociales y ambientales de esa introducción (deforestación, pérdida de fertilidad del suelo, aumento en el uso de agroquímicos con eventuales consecuencias negativas para la población); a la vez que se reconociera su impacto positivo en los ingresos de los productores (fundamentalmente, grandes y medianos) y a nivel de la macroeconomía del país (balanza comercial y recaudación fiscal).

Creemos que este consenso constituye un hallazgo significativo, en particular, considerando los niveles encendidos que alcanzó la controversia en torno a los transgénicos y, en particular, en torno a la soja en los últimos años. Aunque se trata de un consenso inestable y que no deja de lado las diferencias entre las posiciones pro y anti-transgénicos en la Argentina, que siguen tan polarizadas como anteriormente; y aunque no incluya otras cuestiones que quedaron fuera del consenso, puede decirse que sienta las bases de un terreno de discusión común y permite avanzar sobre los problemas identificados por este consenso.

\section{El contexto latinoamericano y la resistencia a las tecnologías}

En América Latina se verifican en estos momentos conflictos de variable intensidad y alcance, que tienen como blanco desarrollos y emprendimientos científico-tecnológicos de magnitud que 
involucran grandes inversiones, convocan a diversos actores y movilizan un amplio espectro de expertises. De la oposición a los transgénicos que comenzó a instalarse a mediados de los noventa y que empalmó luego con las críticas a otro desarrollo agro-industrial vinculado, los biocombustibles; a la resistencia a la producción de pasta de papel; a la oposición a la explotación del petróleo o la minería a cielo abierto; a las represas hidroeléctricas, a la tecnología nuclear, a los tendidos eléctricos, a los gasoductos. Algunos observadores interesados hablan de un florecimiento del ludismo en la región. Dejando de lado la hipocresía en que se basa esta terminología, creemos que la región se encuentra ante un ciclo de protesta ambiental, encendido por una serie de transformaciones vinculadas al proyecto neoliberal de la globalización, y hecho posible por las capacidades desarrolladas por las poblaciones locales en función del ejercicio de la protesta pública, con diferentes variantes de realización (VARA, 2010; VARA, en prensa).

En este trabajo, nos apoyamos en el marco de la "resistencia a las tecnologías", de Martin Bauer, que apunta al derecho de las poblaciones afectadas por la introducción o modificación en el uso de una tecnología, a ser escuchadas en sus preocupaciones y reclamos, y a determinar qué actores responderán ante qué actores por las consecuencias de esa adopción. Bauer explica que, dado que las diversas tecnologías a la vez crean y limitan ciertas oportunidades, es inevitable que las mismas, eventualmente, se encuentren ante la posibilidad de ser contestadas y, en consecuencia, sus promotores deban legitimarlas en el ámbito público. De este modo, Bauer sostiene que en las sociedades democráticas "El control de la tecnología por aquellos que resultan afectados por ella es entonces una agenda deseable" (BAUER, 1995: p. 1).

La historia del siglo XX muestra que, en muchos casos, los grupos sociales afectados lograron hacer valer sus demandas y modificaron el curso de desarrollo y adopción de ciertas tecnologías. En este sentido, la noción de "controversia tecnológica" fue introducida en los tempranos setenta - en coincidencia con los inicios del ambientalismo - para dar cuenta de procesos sociales en 
que distintos actores cuestionaron y afectaron con sus acciones públicas la decisión de actores expertos en relación con la construcción de instalaciones, la incorporación de tecnologías que implican riesgos de salud o ambientales, la aplicación de normativas técnicas que suponen avanzar sobre valores tradicionales o sobre la libertad de elección de los ciudadanos. En estos casos, típicamente, hay actores que promueven el proyecto, actores que lo administran y actores que se oponen; la controversia se suscita debido a las diferentes perspectivas sobre el problema. Mientras que quienes desarrollan un proyecto persiguen un objetivo específico y "trabajan en términos de un cálculo de eficiencia que sólo incorpora costos que pueden ser cuantificados", quienes pueden ver alteradas sus vidas definen los costos "de manera que incluyan los impactos sociales y ambientales” (NELKIN, 1974: p. 3). Por otra parte, corresponde recordar que la bibliografía sobre resistencia a las tecnologías muestra que no abundan los casos en que el conocimiento experto está sólo del lado de los promotores: un ejemplo temprano es el rechazo a la instalación de una central nuclear sobre el lago Cayuga, en el estado de Nueva York, en el que científicos de Cornell University encabezaron la oposición (NELKIN, 1971). Trabajos posteriores confirmaron estas primeras observaciones (NELKIN, 1992).

Una segunda aclaración tiene que ver con la noción de "ciclo de protesta”. En este trabajo, además de las mencionadas líneas bibliográficas de los estudios sociales de la ciencia y la tecnología, vamos a apoyarnos en nociones tomadas del estudio de los movimientos sociales, siguiendo una tradición de diálogo de varias décadas (para una revisión reciente, véase HESS et al., 2008). Por ejemplo, hemos tomado la mencionada noción de "ciclo de protesta”, definida en un trabajo clásico (TARROW, 1993) como "una ola creciente y decreciente de acciones colectivas y reacciones a las mismas cuyas frecuencia, intensidad y formas aumentan y luego declinan de manera cronológicamente aproximada". También tomaremos otras nociones de la bibliografía sobre "política contenciosa”, que integra varias líneas en el estudio de los movimientos sociales (MACADAM; TARROW; TILLY, 2007). 


\section{De la incorporación a la controversia}

Los cultivos transgénicos constituyen una de las tecnologías más resistidas en el mundo, tanto por lo sostenido en el tiempo como por la amplitud y coordinación de la oposición. Algunos aspectos que están en la base de la oposición a esta tecnología en el mundo son la alta percepción de riesgo vinculada con los poderes del ADN, en el marco de la "sociedad del riesgo" que describe Ulrich Beck (1986; ver también SLOVIC, 2000); la desigual distribución de riesgos y beneficios entre consumidores y productores en los transgénicos de primera generación; el desarrollo de esta tecnología por transnacionales, en tiempos en que se promueve y defiende acerbamente el patentamiento de los desarrollos científico-tecnológicos incluso cuando se originan en el sistema académico (RUSE; CASTLE, 2002).

Estos elementos están presentes en las controversias que se han suscitado en América Latina, y se agregan otros: el enfrentamiento entre pequeños y grandes productores, con el antecedente de los latifundios y las siempre pendientes reformas agrarias; el avance sobre tierras de ocupación consuetudinaria, terrenos fiscales y aún reservas naturales; la persistencia de estructuras socioeconómico-políticas cuasi feudales; la debilidad de los gobiernos periféricos y semi-periféricos frente a las empresas transnacionales; la precariedad de los sistemas científicos locales, poco preparados y con poco presupuesto para lidiar con cuestiones de propiedad intelectual y regulaciones para la aprobación comercial de los desarrollos tecnológicos; las inequidades del comercio internacional, en particular los subsidios agrícolas de los países centrales.

Con respecto a la Argentina, un país tradicionalmente agroexportador, puede decirse que la incorporación de la biotecnología agrícola fue inicialmente muy entusiasta. Los transgénicos llegaron al país durante un período de intensificación de la actividad agrícola que se había iniciado en los años setenta y se profundizó en los noventa, cuando fueron superadas dos barreras simbólicas: la superficie de 25 millones de hectáreas destinadas a la agricultura, 
y los 60 millones de toneladas de producción (BEGENISIC, 2002a: p. 2). A pesar de que no todas las reformas estructurales de los noventa tuvieron un impacto positivo en el campo, el proceso, en términos generales, dejó saldos favorables en la productividad total del sector agrícola, donde creció la inversión y se incrementó el uso de agroquímicos y maquinarias. A esto se agregó el aumento de los precios internacionales de productos de exportación tradicionales y no tradicionales de la Argentina (como el trigo, el maíz y la soja) en particular en la primera mitad de los años noventa. La agriculturización del campo argentino y la correlativa disminución de la actividad ganadera tuvo que ver también con la necesidad de incrementar la rentabilidad de las explotaciones, en un contexto en que las actividades agropecuarias no reciben subsidios (TRIGO; CAP, 2003; RECA; PARRELLADA, 2001; RAPOPORT, 2000; BARSKY; GELMAN, 2001).

La soja había sido introducida en el país a comienzo de los años setenta, y su producción fue creciendo paulatinamente. En la década del noventa, la demanda internacional se expandió por la mayor demanda europea primero - entre otras razones, por los acuerdos de Blair House, que implicaron poner un límite a la superficie destinada a oleaginosas en la Unión Europea (LEDESMA, 2003) y luego por la mayor demanda asiática (ABLIN y PAZ, 2001). Desde sus comienzos en el país, resultó ser un cultivo apto para la llamada "siembra de segunda", que seguía a la cosecha de trigo, papel en el que se habían probado otros cultivos de manera infructuosa. A su vez, en esa segunda siembra resultaban apropiadas las técnicas de siembra directa, que implican una mínima labor del suelo pero que requieren una variedad de herbicidas, en particular, en el caso del cultivo de soja en la Pampa húmeda (PEIRETTI, 2001; POVERENE; CANTAMUTTO, 2003). La soja tolerante a glifosato - o soja RR, por Roundup Ready, nombre comercial del herbicida desarrollado por Monsanto que completa el paquete tecnológico resultó una solución muy adecuada para este conjunto de problemas, dado el amplio espectro de acción de este herbicida. Así, la aprobación de este primer transgénico en la agricultura Argentina 
en 1996 y su combinación con las técnicas de siembra directa determinan "un punto de inflexión a partir del cual este cultivo inicia un crecimiento vertiginoso" (BEGENISIC, 2002b: p. 3).

En la temporada 1996/97, las 50.000 ha de soja RR representaban apenas el 0,7 por ciento de la superficie destinada a soja. En 1997/1998, había ya 1.756 .000 ha de soja RR, el 25 por ciento del total. Y en la temporada $2002 / 2003$, se estimaba en unos 12.000.000 ha la superficie destinada a soja, de la cual entre el 95 al 98 por ciento era RR. Tendencia que continúa. La velocidad de adopción de la soja RR en la Argentina resultó inédita incluso en comparación con otras introducciones muy exitosas, como los híbridos de maíz y el trigo con germoplasma mexicano (PENNA; LEMA, 2003). Actualmente, se estima en 19.500 .000 ha el área destinada al cultivo de soja RR, alrededor de la mitad del área sembrada. De hecho, la rápida incorporación de la soja RR hizo de la Argentina el segundo productor de transgénicos del mundo, detrás de Estados Unidos; lugar del que fue desplazada recientemente por Brasil (JAMES, 2010: pp. 40-41).

Hemos analizado (VARA, 2005: cap. 5) que, hasta mediados de la primera década de 2000 , los transgénicos en general y la soja en particular gozaban todavía de una relativa invisibilidad frente a la opinión pública argentina, a pesar de la rapidísima expansión de su adopción en todo el país, y a pesar de que, desde el comienzo de la incorporación de los transgénicos, en la Argentina varios actores anti-transgénicos habían encarado acciones de resistencia; tanto ONGs internacionales - entre ellas, nada menos que Greenpeace, de fuerte actuación en Europa - como organizaciones de movimientos sociales y ONGs locales, como el Grupo de Reflexión Rural (GRR) o el Movimiento Campesino de Santiago del Estero (MOCASE).

Esta situación comienza a cambiar paulatinamente, con dos momentos que marcan puntos de inflexión. El primero tiene que ver con el contexto internacional de una creciente demanda de commodities agrícolas, tanto por el aumento del consumo por parte de países en crecimiento - notablemente, China e India - como por la promoción de los biocombustibles en los países centrales, entre 
otras causas. Panorama que en países productores como Brasil o la Argentina aumentó las presiones para incrementar la producción en busca de crecientes ganancias, exacerbando las tensiones vinculadas a estas dinámicas. De hecho, una de las raíces del grave enfrentamiento entre diversos sectores de los productores rurales y el gobierno argentino que se produjo a mediados de 2008 puede atribuirse a la disputa por la renta extraordinaria originada en la tormenta perfecta que hizo que se dispararan los precios de los alimentos a comienzos de ese año (BARSKY; DÁVILA, 2008: 115-131). En medio de ese enfrentamiento, suscitado en torno al decreto del Poder Ejecutivo Nacional No 125 de ese año, que determinaba la movilidad de los impuestos por exportación para la soja ("retenciones móviles"), por primera vez, un jefe de Estado, la presidenta de la Nación, se refirió de manera pública en términos despectivos hacia la soja transgénica, llamándola una "maleza" que crece casi sin necesidad de cuidados, un hecho que marcó un quiebre en la percepción pública del transgénico estrella.

El segundo momento está relacionado con la difusión pública de un estudio realizado por un prestigioso investigador del sistema público que suponía una fuerte acusación contra la proclamada baja toxicidad del glifosato, de amplísimo uso en la Argentina: el 13 de abril de 2009 el diario Página/12 de Buenos Aires publica una nota basada en una entrevista al doctor Andrés Carrasco, investigador de la Facultad de Medicina de la Universidad de Buenos Aires (UBA) y del Consejo Nacional de Investigaciones Científicas y Técnicas (CONICET), del que fue presidente (ARANDA, 2009). En la misma, Carrasco presenta los resultados de experimentos realizados en su laboratorio de la UBA, entre cuyas conclusiones se sostiene: "concentraciones muy reducidas de glifosato como las usadas aquí respecto de las usadas en el terreno, producen en el embrión efectos reproducibles tanto morfológicos como moleculares acotados" (CARRASCO, s/f: p. 7). En la propia escritura del informe queda claro que el investigador tiene plena conciencia de las implicancias de su difusión. Posteriormente, Carrasco publicaría dicho trabajo junto a su grupo en una revista de toxicología (PAGANELLI et al., 2010). 
A partir de entonces, la resistencia relativamente sorda que había existido contra los transgénicos en la Argentina, gana una magnitud tal que puede hablarse ya de la emergencia de una auténtica controversia tecnológica pública, con capacidad de impactar en los planes de los promotores (ARANCIBIA, en prensa).

\section{Cierre del marco regulatorio: riesgo biofísico y mercados externos}

Con respecto a la conformación del marco regulatorio de la biotecnología agrícola en la Argentina y la cuestión de su "cierre" en torno a la regulación de los riesgos biofísicos, hay consenso en los actores entrevistados en cuanto a que estas decisiones se debieron a la confluencia de intereses de las empresas transnacionales de agroquímicas y semilleras y sectores locales, preocupados por la competitividad de la producción agrícola argentina. Desde el punto de vista conceptual, el entrevistado D explica la orientación del marco regulatorio, focalizado en el riesgo biofísico y el principio de equivalencia sustancial:

El marco regulatorio [argentino] es un marco de tipo preventivo, lo que trata es de evitar impactos negativos sobre el sistema en el cual se introduce el material. Lo que se parte es de una hipótesis de riesgo, ver si ese material puede causar algún tipo de daño. Y en realidad lo que se hace es compararlo con el material convencional, con el que no ha sufrido el cambio. (Entrevistado D)

Quien introduce otra motivación para la creación del marco regulatorio en la Argentina es el entrevistado B, director de una empresa argentina dedicada a la consultoría sobre negocios agrícolas, autor de numerosos estudios sobre el impacto de la introducción de transgénicos en la Argentina, quien alude a un caso denunciado de pruebas experimentales realizadas por el Wistar Institute de Filadelfia conjuntamente con la Organización Panamericana de la Salud con un virus de la rabia bovina modificado por ingeniería genética, sin informar ni solicitar autorización de las 


\section{Biotecnología agrícola y "sojización" en la Argentina: controversia pública... Ana María Vara • Agustín Piaz • Florencia Arancibia}

autoridades locales, a mediados de la década del ochenta en la localidad bonaerense de Azul (JOYCE, 1986; CONNOR, 1988). Se trata de una explicación bastante extendida:

(...) creo que ahí se produjo la noción de un vacío regulatorio, de algo nuevo que estaba apareciendo y que no tenía marco apropiado —ni acá ni en ningún otro lado— - y entonces se tomaron decisiones con un sentido, no diría precautorio, sino como algo que debería ser reglamentado para asegurarse que se cumplieran ciertas normas... (Entrevistado B)

Ahora bien, en relación con actores internacionales, el interés de la empresa transnacional Nidera por introducir variedades transgénicas, especialmente de soja, en la Argentina, es destacado por el entrevistado D como otra motivación para introducir el marco regulatorio. Tras comentar que la soja RR comenzó a ensayarse en la Argentina en 1991, es decir, el mismo año de la creación de la CONABIA, comenta:

En la Argentina lo que se hizo es trabajar en programas de mejoramiento genético sobre las variedades que se usaban en el país, porque si no, es como extrapolar algo que puede ser útil en el hemisferio norte pero no es útil acá. Entonces lo que se hizo y eso lo hizo bastante, el que trabajó mucho en el desarrollo en las soja que se usa es Rodolfo Rossi [de Nidera], que es un ingeniero agrónomo que es el que fue el padre del desarrollo mediante un programa de mejoramiento genético de la soja RR. (Entrevistado D)

Más adelante en la entrevista, el entrevistado D menciona otras empresas interesadas, del mismo modo que reitera que había en el país capacidad científica en las áreas pertinentee: "Y aparecieron tres empresas, Nidera, lo que ahora es Ingenta pero que en ese entonces era otra empresa, y Ciba Geigy y querían presentar un maíz, una soja y un algodón, eso fue el inicio..." En subrayar el interés de Nidera por los ensayos a campo en la Argentina también concuerda el entrevistado $\mathrm{C}$, director de asuntos regulatorios para Argentina de una empresa transnacional de semillas y agroquímicos: "El sistema se estableció como una respuesta de la entonces Secretaría de Agricultura a una necesidad que emergía, principalmente 
desde el área de investigación. Algunas compañías nacionales de la época como Nidera, apoyaron fuertemente la creación de este marco regulatorio, para poder desarrollar tareas de investigación en nuestro país". Es de destacar que el entrevistado C califica de "nacional" a Nidera, una empresa semillera transnacional con base en Holanda. En esta estrategia de insistir en presentar la creación del sistema regulatorio como una iniciativa en la que estuvieron fuertemente involucrada actores argentinos coincide con el entrevistado $\mathrm{D}$, vinculado al sistema regulatorio.

En relación con el cierre temprano del marco en torno a cuestiones de bioseguridad, son elocuentes las declaraciones del entrevistado E, experto en genética y biotecnología del Instituto Nacional de Tecnología Agropecuaria de la Argentina (INTA) y asesor de CONABIA. Sin embargo, el entrevistado E destaca la mencionada peculiaridad del sistema argentino: la consideración de si la aprobación de un evento transgénico puede afectar el comercio exterior de la Argentina, que excede las cuestiones de riesgo biofísico, ya que tiene que ver con aspectos vinculados al comercio exterior. Es significativo que el entrevistado E considere que esa orientación hacia el comercio exterior, en realidad está pensada para proteger las ventajas económicas de la exportación para la población argentina: "Esas normas] Consideran el mercado extranjero pero en relación con la ecuación interna. Si Argentina mete un evento que después se va a mezclar con todo lo demás, desde mi entendimiento - que no soy un experto - lo que hace es velar por la ecuación general de la sociedad".

El hecho de que la palabra final sobre la aprobación de un transgénico en la Argentina la tenga la Dirección Nacional de Mercados Agroalimentarios y que la misma base su criterio en aspectos que tienen que ver con cómo el mismo podrá afectar el comercio exterior es un punto significativo, que distingue al sistema regulatorio argentino de otros que no están tan orientados a la exportación, como el norteamericano. De hecho, es precisamente debido a la última etapa de revisión de la Dirección de Mercados 
que la Argentina pudo seguir la llamada "política espejo", aprobando sólo los eventos ya aprobados en Europa, política que le permitió seguir exportando al bloque europeo sin trabas, pese a la moratoria de facto iniciada en 1998, que sí afecto las exportaciones norteamericanas y que derivó en un reclamo de los Estados Unidos, Canadá y la Argentina ante la Organización Mundial de Comercio (VARA, 2004).

En el mismo sentido de valorizar en términos de beneficios para la sociedad argentina este aspecto del sistema regulatorio nacional, comenta el entrevistado D: "Los impactos sociales no forman parte del sistema regulatorio de bioseguridad; el impacto social en realidad el único país que tiene algo que se asemeja a un impacto - pero que es comercial, por el momento - es la Argentina”. Más adelante, reconoce que el sistema regulatorio no tuvo en consideración los impactos sociales, ni los pequeños productores: "Es una cosa en la que no se pensó, el contexto socioeconómico, las regulaciones son regulaciones que analizan el evento, no el sistema de producción".

Ahora bien, con respecto al sentido de la creación del marco regulatorio en función de la incorporación de la tecnología de transgénicos, son varios los entrevistados que destacan la percepción temprana de que esta tecnología podría beneficiar a la Argentina en tanto que país agroexportador. Así lo sostiene el entrevistado L, funcionario de la Cancillería Argentina, quien comenta que la visión de las oportunidades ya estaba presente a comienzos de los noventa. ${ }^{2}$ En el mismo sentido de destacar la importancia del sector agropecuario en la economía argentina y sus posibilidades competitivas se pronuncia el entrevistado A, miembro de una asociación argentina vinculada a la industria biotecnológica y dedicada a la promoción de esta tecnología: "Porque iba a ser una tecnología que iba a tener una aplicación directa en la agricultura y, de acuerdo con nuestro perfil y la importancia de la agricultura

2 No disponemos de declaraciones textuales del entrevistado L porque, si bien se prestó a la entrevista, se opuso a que la misma fuera grabada. 
en la Argentina y en los cultivos en los que se estaban introduciendo estas características, hacían pensar que Argentina iba a tomarla desde el vamos". También el entrevistado E comenta que la biotecnología agrícola podía favorecer la competitividad argentina, y que requería un marco regulatorio específico: "La aparición de esta tecnología y su llegada al mercado agropecuario fue percibida desde aquí como una ventana de oportunidad (...)".

Lo que resulta significativo es que entrevistados enlistados en el polo anti-transgénicos coinciden en atribuir el origen del marco regulatorio argentino a la cuestión de la competitividad argentina por la incorporación de la biotecnología agrícola y, más importante aún, lo valorizan explícitamente. Es lo que hace el entrevistado G, coordinador de un programa vinculado a la problemática de la deforestación en la sede argentina de una ONG ambientalista internacional, comparando la situación de la Argentina con la de países que no lograron establecerlo antes de la incorporación de los transgénicos por parte de sus productores agrícolas (parece estar refiriéndose a Brasil):

Argentina venía siendo un importante productor de soja, desde antes de la introducción de los transgénicos y con una industria de procesamiento de soja también importante. Había un conglomerado sojero que ya tenía una posición importante en Argentina y, con la introducción de los transgénicos, vieron que era claramente el lugar hacia donde querían ir y se posicionaron, afortunadamente bien, con un marco regulatorio para poder incorporar rápidamente esa tecnología en la Argentina. Digo 'afortunadamente' porque en otros países que también tenían un sector sojero importante fue al revés. Lo importante es que en el caso de la Argentina primero apareció el marco regulatorio y después se introdujeron los transgénicos. (Entrevistado G)

El entrevistado $G$ también menciona y valoriza las ventajas agronómicas, en términos de protección del suelo, que permitió la adopción de eventos con resistencia a herbicidas, particularmente la soja tolerante a glifosato, en función de la incorporación de la siembra directa: "Es una tecnología que permite cultivar sin arar el suelo. Gracias a eso se solucionaron un montón de problemas de 
erosión, de conservación del suelo, que eran problemas serios en Argentina y que hoy siguen existiendo pero son más moderados".

Ahora bien, los aspectos centrales del marco regulatorio argentino, es decir, los referidos a las cuestiones de riesgo biofísico, están en discusión por parte de los propios actores que promovieron su cierre: entrevistados del frente pro-transgénico se han referido a la relativamente nueva problemática de los eventos apilados - más de un evento en un mismo cultivo - y a la necesidad de simplificar la regulación, incluso, separando a la Argentina de las exigencias europeas.

El entrevistado D se refiere a la cuestión de los eventos apilados como un tema que se presenta en el horizonte inmediato: "ahora pueden aparecer variedades que son muchos más complicadas desde el punto de vista del funcionamiento, y aparecen en cuestión todos los eventos que se denominan apilados, cuando tenés dos caracteres que se combinan por cruzamiento. Ahí tenés otro tema que es de discusión regulatoria".

Con respecto a la posible baja de regulación, el entrevistado B relaciona esta cuestión con una puesta del día del marco regulatorio, a partir de un aprendizaje de las aprobaciones de eventos ya realizados: "yo no digo que relajemos los mecanismos que tenemos, porque no soy especialista, lo que digo es que el sistema no tiene un mecanismo de aprendizaje de la realidad, de incorporación de nueva información que permita evolucionar junto con la tecnología"

El entrevistado D retoma una cuestión presente hace tiempo en la argumentación sobre la alta regulación (y sus correlativos altos costos) que debió incorporar la Argentina, como una limitante para el propio desarrollo científico local y su orientación a problemáticas locales: "Hay ciertas cosas que se adaptan muy bien a problemas locales, que son los desarrollos que vienen. Trabajemos en tecnología para desarrollos locales". En este sentido, un biotecnólogo como el entrevistado $\mathrm{F}$ manifiesta una opinión positiva con respecto a que la Argentina se separe de las exigencias de la regulación europea, y expresa su optimismo con respecto a estos eventuales cambios. En términos del comercio exterior, lo vincula con nuevos compradores 
de los productos argentinos, entre ellos, China: "Y eso se va a producir en breve, se tiene que producir. En la balanza comercial de la Argentina, Europa cada vez pesa menos. Nuestra producción está yendo a China e India, a los países árabes".

Ahora bien, estas opiniones a favor de reducir los requisitos de riesgo biofísico del marco regulatorio argentino tienen, obviamente, un peso limitado. Está claro que la Argentina, como país exportador, no puede imponer por sí misma esos cambios, salvo que, como sugiere el entrevistado $\mathrm{F}$, se aparte de las exigencias del mercado europeo, apoyándose en otros mercados. Lo cual no parece muy probable en este momento aunque, ciertamente, es un punto que merece ser investigado con más profundidad. Las opiniones recabadas pueden ser más relevantes para predecir la posición de la Argentina en la arena internacional en función de estas discusiones, en particular considerando que las mismas son sostenidas por actores vinculados o cercanos a las instituciones regulatorias. En ese aspecto, sin embargo, la predicción en términos de la posición internacional de la Argentina no resultaría muy novedosa: está en los intereses de un país agroexportador como la Argentina que las regulaciones sean bajas, mientras no pongan en riesgo la producción.

\section{Reapertura informal del marco regulatorio}

Si bien, como acabamos de ver, el marco regulatorio de la biotecnología agrícola en la Argentina se cerró tempranamente en torno a la regulación de los riesgos biofísicos, orientándose a la rápida incorporación de una tecnología que se consideraba importante en función de la competencia internacional por el mercado de commodities agrícolas, lo cierto es que la resistencia de diversos sectores, que derivó en la presente controversia, ha logrado reabrirlo periódicamente de manera informal, a través de regulaciones complementarias, obligando a las autoridades involucradas

- que superan las estrictamente encargadas del marco regulatorio 
de esta tecnología - a tener en cuenta aspectos que fueron o bien dejados de lado inicialmente, o bien no fueron contemplados por resultar poco previsibles.

En las entrevistas realizadas surge, en primer lugar, la mención de una normativa para controlar el uso de la soja como alimento para niños y sectores vulnerables. En respuesta a la campaña Soja Solidaria, que consistía en la donación de soja con destino a comedores populares y fue impulsada en torno a la crisis de 2001 por la Asociación Argentina de Productores de Siembra Directa (AAPRESID), fuerte promotora de los transgénicos (VARA, 2005: cap. 5), comenta el entrevistado $\mathrm{H}$, fundador de una organización de movimiento social argentina dedicada a cuestiones rurales, que su agrupación logró reunir a funcionarios del sistema de seguridad social para discutir sobre la calidad nutricional de la soja. Como resultado, el Consejo Nacional de Coordinación de Políticas Sociales (2003) emite el documento Consideraciones sobre la soja en la alimentación. Sus conclusiones incluyen recomendaciones acerca de que la soja no es un buen alimento para menores de cinco años; que "se puede considerar" que la soja y el bebible de soja están contraindicados para menores de dos años; que la soja no debe usarse como sustituto de la carne ni de la leche; que no debe considerarse "como un elemento central" de la alimentación de personas sanas ni de personas desnutridas.

Una segunda línea normativa tiene que ver con el problema de la deforestación, en que resultó clave la aprobación de la Ley de Presupuestos Mínimos de Protección Ambiental de los Bosques Nativos No 26631 o "Ley de Bosques" en 2007, destinada a controlar la deforestación originada en el proceso de agriculturización rápido y desordenado; también puede decirse que tiene objetivos sociales, en particular referidos a las poblaciones nativas. Sostiene el entrevistado $\mathrm{G}$ atribuyendo a su agrupación la iniciativa de la ley:

No nos oponemos a que crezca la producción de soja o las exportaciones de la Argentina, pero sí creemos que tenemos que controlar que ese crecimiento no afecte a los pueblos originarios, a los pueblos campesinos, a los bosques, al agua, etc. Así que lo que 
en principio promovimos fue la sanción de la Ley de Bosques, que se sancionó a fines de 2007, que lo que pide es que cada provincia haga un ordenamiento ambiental de sus bosques. (...) Entonces se le pone un límite geográfico, se dice dónde se puede avanzar y dónde no, ya sea con la soja, la ganadería o cualquier otro cultivo. (Entrevistado G)

De esta cita, destacamos la aceptación por parte del entrevistado $\mathrm{G}$, un actor que puede considerarse anti-transgénicos, de que la deforestación no se debe únicamente a la incorporación de transgénicos en general, ni a la soja transgénica en particular; que consideraría aceptable el crecimiento de la producción y exportación de soja en la Argentina; y el reconocimiento, al final, de que determinadas actividades producen una rentabilidad que, de perderse debido a la aplicación de la ley, debería ser compensada por otras actividades.

En un sentido similar habla de la Ley de Bosques el entrevistado I, coordinador de un programa dedicado a clima y deforestación en la sede argentina de una ONG ambientalista internacional y enrolado también en el polo anti-transgénicos, sosteniendo que dicha ley no es específica para la soja sino que busca ordenar el proceso de agriculturización; si bien su valoración de este hecho es negativa: "lo que sí se hizo fue ir imponiendo leyes, como la Ley de Bosques, que impiden la expansión de la agricultura para no afectar un ecosistema como el bosque, pero no la expansión agrícola de tal producto, algo que podría existir tranquilamente".

Más interesante todavía resulta la apreciación sobre la Ley de Bosques del entrevistado A, que se enlista entre los actores pro-transgénicos. Su cita deja de manifiesto en qué medida aún actores pro-transgénicos admiten el impacto de la agriculturización orientada a la soja en la deforestación. Se pregunta el entrevistado A: “¿Cuál es el punto? ¿Está bueno talar árboles nativos? No, ni para eso ni para un camino, ni para un shopping, ni un aeropuerto, para nada. Ni para soja. Entonces, es una política sobre bosques, digamos". Vale retener la última afirmación, referida a que la Ley de Bosques no es una regulación sobre transgénicos sino sobre deforestación. 
Otros actores pro-transgénicos muestran asimismo su aceptación de que la deforestación está relacionada con la incorporación de la soja transgénica aunque no exclusivamente, en términos similares a las citas de los entrevistados G e I. Se permite dudar el entrevistado B: "Decimos que [la soja] avanza sobre el bosque... probablemente sí. ¿Realmente avanza sobre el bosque? Hay datos que me hacen dudar, porque la soja no se hace en ladera. ¿Avanza sobre el bosque o sobre zonas del bosque degradadas, planas, donde había ganadería extensiva y reemplaza a eso?".

La entrevista a $\mathrm{F}$, uno de los científicos más encolumnados en la defensa de los transgénicos en el debate público, tiene tramos donde atribuye la responsabilidad de la deforstación a la soja transgénica de manera aún más asertiva. En primer lugar, destaca las cualidades agronómicas del paquete de soja con glifosato y siembra directa, que permitió plantar soja donde era previamente muy difícil, cuestión significativa porque hace al corazón de esta tecnología, al ser específico de la misma: "Por ejemplo, había zonas donde no se podía sembrar soja por las malezas. Necesitabas tanto herbicida y tanta guerra química... Con el paquete de transgénicos tolerantes a herbicidas más la siembra directa, se hizo posible que dos o tres millones de hectáreas en Argentina entraran en producción. Eso es conocido." El cierre de la cita parece apuntar a que el entrevistado $\mathrm{F}$ tiene conciencia del consenso generado sobre este punto.

Finalmente, una cita del entrevistado D deja en evidencia el esfuerzo por alcanzar un delicado equilibrio en la atribución de responsabilidad a la soja transgénica por la deforestación: "A veces es más fácil echarle la culpa a una tecnología que hacerse responsable del sistema como tal (...). He visto en la década del 80 en algún lugar con las topadoras tirar árboles de 40 metros de altura y el suelo que te queda es un suelo suelto que no está acostumbrado a tener un tapiz arbóreo como corresponde. La soja transgénica no existía, ahí hay un problema de otro tipo".

De estas apreciaciones surge un punto clave de consenso entre actores pro y anti-transgénicos: que la agriculturización tuvo 
como impacto la deforestación, y que ese problema debe ser atendido. Y, del mismo modo, coinciden en que no se debe responsabilizar sólo a los transgénicos ni a la soja transgénica. Por otra parte, unos y otros valorizan la Ley de Bosques y la consideran una posible solución al problema.

De nuestras entrevistas surge una tercera línea normativa, que busca atender a la problemática del uso de agroquímicos; y que está en una etapa previa a la alcanzada con la sanción de la Ley de Bosques. Los entrevistados coinciden en que está en una etapa de discusión, aunque difieren en cuanto a la estimación de si puede o no conformarse de modo definitivo.

El entrevistado I sostiene que hay estudios parciales sobre la cuestión del uso de agroquímicos y sus impactos en la salud de los usuarios y las poblaciones aledañas, pero que deberían hacerse estudios integrales. Su idea de cómo regular esta cuestión parece apuntar hacia algún tipo de macro-regulación, que supere los fallos judiciales de alcance local, que ha sido la tendencia en los últimos años para enfrentar el problema. Sin embargo, no parece optimista en cuanto a que esa normativa pueda alcanzarse ni en cuanto a que esos impactos puedan controlarse eficazmente: "Los estudios son todos parciales, en lugares específicos, pero no hay estudios integrales. (...) En el tema salud no se puede simplemente decir que hay un problema, sino que hay que solucionarlo y la solución quizás no existe”.

Sin embargo, otros entrevistados confían en la posibilidad de que se alcance algún tipo de legislación más comprehensiva. El entrevistado $\mathrm{K}$, diputado nacional por un partido de centro-izquierda, propone una ley que limite las fumigaciones para no afectar la salud humana ni otras actividades económicas: "Lo que estamos proponiendo es limitar a 1000 metros alrededor de los pueblos, y ver cuáles son las producciones que se pueden hacer en ese territorio para determinar los planteos que se pueden llevar al Estado, al Congreso respecto a ese territorio" (...). Dos agroquímicos, ambos relacionados con el cultivo de soja transgénica, surgen como preocupaciones: glifosato y endosulfán. Sostiene el entrevistado K comparando ambos: 


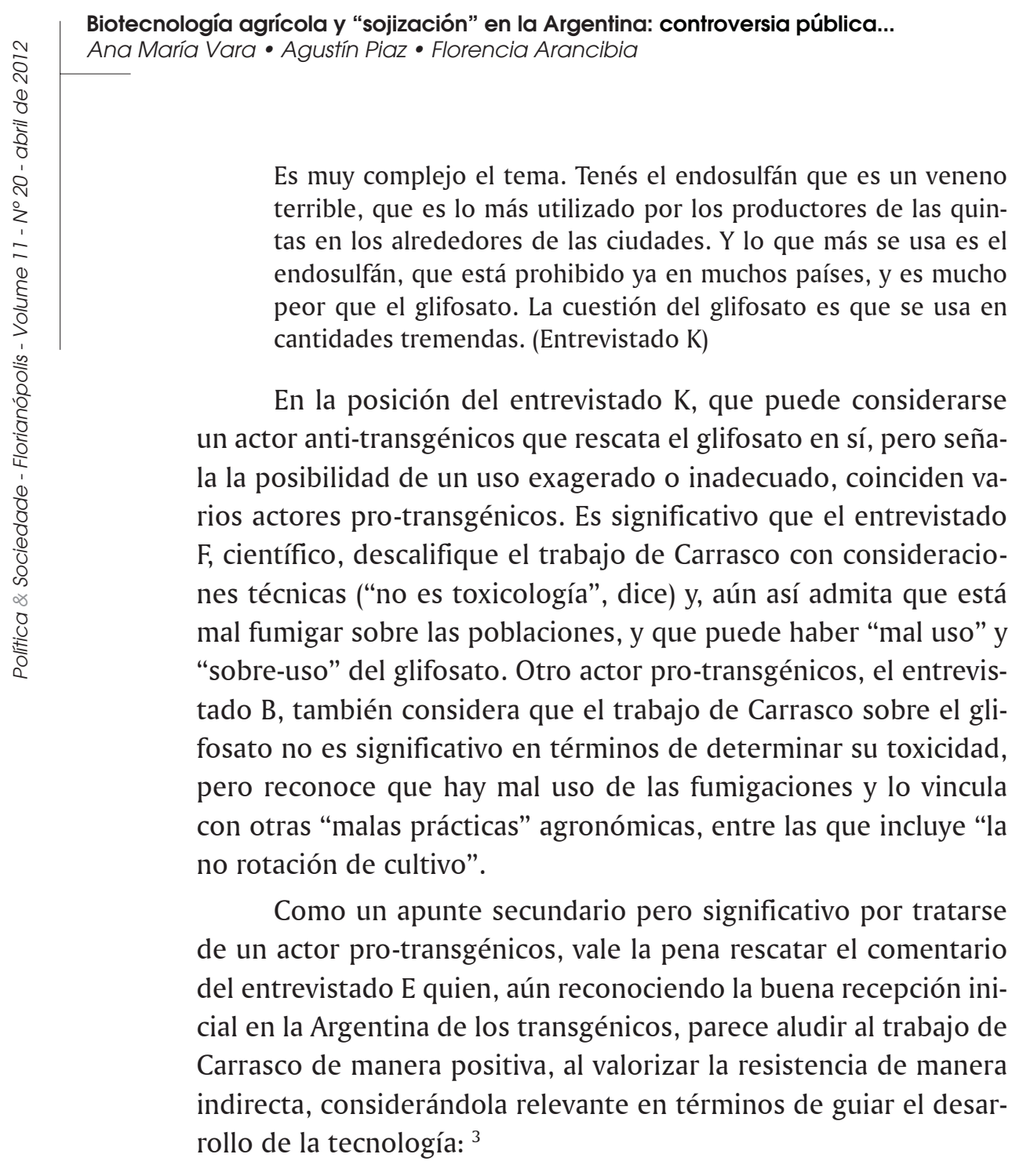

Si hoy el 99,99 por ciento de la soja es transgénica, más del 90 por ciento del maíz es transgénico y más del 70 por ciento del algodón es transgénico, quiere decir que en términos generales ha habido una sociedad receptiva a esa tecnología. Afortunadamente, no todos piensan lo mismo, hay gente que levanta la voz en un sentido

3 Se trata de una visión funcionalista de los fenómenos de resistencia, muy próxima a la noción de "dolor organizacional", que se basa en una analogía que pone el acento en "su capacidad para cambiar la actividad en beneficio del futuro de la actividad" (BAUER, 1995: p. 403). 
distinto y tendrá la responsabilidad y la enorme tarea de mostrarle a la sociedad que eso no es conveniente... es esa diversidad la que asegura que no metamos la pata. (Entrevistado E)

De manera previsible, un actor anti transgénicos como el entrevistado $G$ dedica más detalles a la consideración del uso del glifosato. Es significativo, sin embargo, que él también destaque la cuestión del mal uso del glifosato y no lo condene en sí mismo:

Ahora el glifosato no es tan efectivo como antes, así que los productores empiezan a usar más de lo que dice la etiqueta, ya no alcanza y usan más. La etiqueta señala que el producto tiene una toxicidad tal cuando se aplica en tal proporción, pero cuando se aplica más no se sabe. Y tampoco hay muchos estudios sobre cuáles son sus efectos en los organismos del suelo, el agua. Aparecen estudios de que tiene efectos en la salud de la gente, encima en Argentina hay muchos casos de fumigaciones cerca de viviendas, pueblos, que no es para lo que está hecho el glifosato, que se tiene que aplicar en cultivos. Ahí se dice que tiene una toxicidad baja, pero si se aplica cerca de un pueblo donde hay niños, embarazadas, eso no se estudió... o se estudió pero hay controversias sobre cuáles son los efectos. Así que la preocupación es sobre el glifosato, sobre todo teniendo en cuenta que la adopción es masiva y a gran escala. (Entrevistado $\mathrm{G}$ )

Cercanas a estas apreciaciones se encuentran las del entrevistado I, también de una ONG ambientalista internacional, quien habla asimismo de la resistencia de las malezas, del aumento de las concentraciones del glifosato, y de la amplitud de la superficie involucrada, pero no condena de plano al glifosato:

Lo que pasa es que es compleja la situación, porque cada vez las malezas resisten más. Pero la misma gente del campo señala que antes usaba herbicidas mucho peores que el glifosato. Lo que pasa es que hay muchas malas prácticas, fumigaciones sobre pueblos, niños, banderas [banderilleros], etc. Tampoco hay estudios hechos sobre el impacto de rociar 1000 millones de litros de glifosato sobre 17 millones de hectáreas. (Entrevistado I)

Es notable que el entrevistado $\mathrm{M}$, un científico que asesora a la organización de movimiento social de orientación rural, no 
se concentre en su argumentación sobre la toxicidad del glifosato sino en el sobre uso y el mal uso, coincidiendo en este punto con los entrevistados pro-transgénicos $\mathrm{F}$ y $\mathrm{B}$, además de con los entrevistados anti-transgénicos $\mathrm{K}, \mathrm{G}$ e I:

Cuando se lanzó esto era como un experimento. Entiendo que era imposible predecir 20 millones de hectáreas con 200 millones de litros de pesticidas, etc. De los cuales 180 son sólo de glifosato. Nunca tenés todas las variables, lo único que podés hacer es avanzar despacio, monitorear y parar cuando alguna luz amarilla se prenda. Estos son territorios liberados. Donde se recomiendan 2 ó 3 litros por hectárea se usan 12. (Entrevistado M)

Nuevamente, entre los entrevistados hay quien se atribuye la responsabilidad de haber movilizado a actores clave y de haber hecho cambiar la percepción pública: el entrevistado J sostiene haber realizado una recopilación bibliográfica que habría inducido a Carrasco a hacer su investigación.

Y sacando de foco la cuestión del glifosato, el entrevistado $\mathrm{H}$, de la misma agrupación que $\mathrm{J}$, sostiene que el problema no es el glifosato en sí, dado que Monsanto lo puede reemplazar con el otro herbicida, el dicamba, sino el "modelo", basado en transgénicos, que considera es un "experimento" realizado en 20 millones de hectáreas, así como considera un "experimento" el hecho de que la población argentina coma transgénicos, alimento al que atribuye un deterioro generalizado en el sistema inmunitario de los argentinos. Es decir, introduce cuestiones alimentarias que han quedado fuera del consenso.

También se aparta del consenso que el reconocimiento del uso exagerado del glifosato resulte un argumento para que un entrevistado pro-transgénicos, como $D$, se exprese a favor de la incorporación de nuevos transgénicos que permitan superar este problema: "La idea es siempre sigas investigando y que no te quedes con el evento inicial; fijate que el evento 40-32, la soja RR, es un evento viejo ahora, en realidad uno tiene que tener renovación para evitar la resistencia (...)". 


\section{Conclusiones}

En Argentina, la introducción de la biotecnología agrícola coincidió con una intensificación de la producción agrícola en detrimento de la ganadería, y con el aumento de la producción de soja, así como con la orientación de parte de esa producción a la provisión de biocombustibles. No es el caso de Estados Unidos, donde el maíz transgénico también tuvo un gran desarrollo y donde también ocupa un lugar importante en relación con los biocombustibles. Algo similar puede decirse de Brasil, aunque con mayor inclinación a la sojización al estilo argentino, pero con una diferente realización en términos de biocombustibles, ya que en este aspecto ha hecho una fuerte apuesta, histórica, en la caña de azúcar. Entonces, puede decirse que como rasgo característico, único, del proceso de incorporación de biotecnología agrícola en los noventa en la Argentina, se da una situación en que transgénicos es sinónimo de agriculturización, de sojización y de biocombustibles.

Esta peculiar forma que adoptó la adopción de la biotecnología agrícola en la Argentina determina que debates que en otros contextos nacionales aparecen como relativamente diferenciados se vean superpuestos y, hasta cierto punto, confundidos, en este país. Otra consecuencia es que, dada la enorme superficie afectada por estas transformaciones, parecería que no pudiera haber debate vinculado con las actividades rurales que no toque la cuestión de la soja transgénica. Sin embargo, sinonimia no supone identidad, de modo que es posible en el debate público y en las normativas establecer diferenciaciones.

En este trabajo hemos analizado cómo se conformó y se cerró el marco regulatorio de la biotecnología agrícola en la Argentina, concentrándose en cuestiones de riesgo biofísico y sumando cuestiones que tienen que ver con el comercio exterior. Asimismo, hemos hallado que, pese a ese temprano cierre, el marco regulatorio fue abriéndose de manera reiterada, respondiendo a distintas demandas a través de la conformación de una extensión informal del mismo, que fue complementando las cuestiones de riesgo 
biofísico y de protección de las exportaciones con otras normativas, como la referida al uso de la soja transgénica como alimento para grupos o sectores vulnerables; la destinada a contener el proceso de deforestación inducido por la agriculturización marcada por la intensificación de la producción de la soja transgénica; y la destinada a regular el uso de ciertos agroquímicos, actualmente en discusión.

De acuerdo a las entrevistas realizadas, esta extensión informal del marco regulatorio fue aceptada tanto por actores anti-transgénicos, que de algún modo participaron en la resistencia que llevó a la instauración de esa normativa, como por actores pro-transgénicos. De estos últimos puede decirse que su aceptación pudo deberse a que esta normativa complementaria no está dirigida específicamente a la tecnología, sino a algunos de sus impactos que, por otra parte, no se le atribuyen en exclusividad. Éste es el punto clave: la Ley de Bosques no es para controlar los transgénicos o la soja, sino la agriculturización. Algo similar puede decirse con respecto a que las normativas sobre las fumigaciones están destinadas a controlar los agroquímicos, no específica o únicamente el glifosato.

Así como la extensión informal del marco regulatorio de la biotecnología agrícola en la Argentina puede atribuirse a los fenómenos de resistencia, que derivaron en la constitución de una controversia tecnológica, lo mismo puede decirse de la emergencia de un cierto consenso entre actores pro y anti-transgénicos incluyendo a representantes del propio sistema regulatorio, con respecto a algunos impactos negativos sociales y ambientales de la introducción de transgénicos. Otro componente importante de ese consenso es el reconocimiento de los beneficios que deja la soja transgénica a los productores argentinos y a la macroeconomía del país; si se quiere, un punto a favor de los actores pro-transgénicos. Ahora bien, entre los reclamos que quedaron fuera de ese consenso se cuentan otras demandas en relación con el riesgo alimentario, que ya tienen cierto tiempo. En sentido opuesto, también actores protransgénicos buscan modificar el marco regulatorio, en este caso el formal, reduciendo los requisitos de aprobación de los eventos. 
Puede decirse, entonces, que es previsible que la controversia actual en torno a los transgénicos continúe en la Argentina, tanto porque esta tecnología probablemente siga expandiéndose, se persista en tratar de bajar la regulación formal e incorporar nuevos eventos ${ }^{4}$ de un lado; como por el hecho de que los actores que la resisten han tenido éxitos parciales y tienen importantes recursos para continuar la protesta, del otro. Además de las organizaciones de movimiento social y los expertos que producen estudios para apoyar los reclamos, es importante que esta controversia coincide con un ciclo de protesta ambiental en América Latina, lo que permite la articulación con otras resistencias. Es significativa, asimismo, la disponibilidad de discursos apropiados para encuadrar la controversia en marcos interpretativos de amplia aceptación, tanto de tipo ambiental clásico - y, por lo tanto, de origen en los países europeos o en Estados Unidos - como específicos de la región: notablemente, el que en trabajos previos hemos dado en llamar "contra-discurso neocolonial de los recursos naturales". Este contra-discurso facilita la articulación con protestas vinculadas a otras tecnologías, como contra la industria de la celulosa y del papel, contra la minería, contra la explotación de petróleo entre otros; y no sólo en la Argentina sino, más en general, en América Latina.

Este contra-discurso tiene una matriz narrativa que vincula la explotación de los recursos naturales con la explotación de poblaciones vulnerables, por parte de actores extranjeros aliados con socios locales. A los explotadores extranjeros se les atribuye una codicia insaciable, y se describen sus acciones como "saqueo", expolio", "depredación"; mientras que a los socios locales se les atribuye complicidad con la explotación; sus acciones se describen como "vender" o "entregar". Asimismo, ese contra-discurso cuenta la historia de

4 Al terminar este artículo, se discutía el anuncio sobre un evento de resistencia a la sequía, el HAHB-4 desarrollado por investigadores argentinos y licenciado a una empresa norteamericana, que fue incorporado a soja, trigo, maíz y alfalfa. Greenpeace denunció que el mismo "implicará el fin de los últimos bosques nativos chaqueños, ya que permitiría realizar agricultura en suelos boscosos donde llueve muy poco y que poseen suelos salitrosos" (citado en "La semilla que promueve el desmonte", 2012). 
Biotecnología agrícola y "sojización" en la Argentina: controversia pública...

Ana María Vara • Agustín Piaz • Florencia Arancibia

América Latina como dos etapas de explotación por parte de actores extranjeros, similares ambas pese a sus aparentes diferencias: durante el período colonial, marcado por la apetencia de metales preciosos; y durante el período de independencia, situación que consideran de dependencia neocolonial, marcada por la apetencia por otros recursos naturales, como los agrícolas o, más recientemente, el agua (VARA, 2009: Introducción).

Entre los entrevistados, tres actores anti-transgénicos apelado de manera clara a este marco interpretativo maestro en su discusión sobre este tema: los entrevistados M, K e I. En el caso del entrevistado $\mathrm{M}$, se trata de una apelación completamente consciente y reiterada, que puede observarse en sus apariciones públicas y sus escritos de divulgación (que no citaremos para no poner de manifiesto su identidad). En la entrevista realizada, sostuvo, vinculando la cuestión de los transgénicos con la minería a cielo abierto, otra tecnología fuertemente resistida en la región (VARA, 2010), y en relación con el neoliberalismo, es decir, aludiendo a políticas impuestas desde fuera:

Bueno, para mí tiene que ver con lo que significa lo que es el modelo neoliberal extractor de beneficios de estos países. Creo que es muy claro. Vos tenés algún tipo de producción como la minería a cielo abierto que claramente es destructiva del ambiente, extractivo absolutamente, que no sirve para mejorar la calidad de vida de nuestros habitantes y que genera enormes niveles de ingreso a sectores muy concentrados; para nosotros es parte del modelo neoliberal, y no atacarlo, es no atacar de fondo el corazón del modelo neoliberal. (Entrevistado M)

El entrevistado K, por su parte, establece una clara distinción entre actores con los que sostiene que está dispuesto a discutir en busca de soluciones, que son locales; y actores a los que se opone, que son extranjeros y a los que califica de abusivos. Ciertamente, es una distinción afín con el contra-discurso neocolonial de los recursos naturales:

La principal controversia es entre los ambientalistas y los productores, porque las controversias con la multinacionales, los pooles 
de siembra, Monsanto y Cargill, están tan claras que no queremos saber nada, no nos parece un controversia, nos parece un abuso. Yo con los fumigadores aéreos sí tengo una controversia, discuto, pero yo a Monsanto, Cargill los quiero acabar. (Entrevistado K)

La semejanza de la situación presente con la situación colonial, otro aspecto clave del contra-discurso neocolonial de los recursos naturales, aparece en una cita del entrevistado I, cuando se refiere a la exportación de soja por parte de la Argentina: "Sí, 200 años después está haciendo lo mismo que cuando era una colonia que recién arrancaba, encima exporta materia prima que no genera valor agregado".

Quisiéramos destacar dos puntos más, como cuestiones emergentes en el momento en que se realizaron las entrevistas: la cuestión de la propiedad de la tierra en la Argentina y su "extranjerización”, y la creciente relación con China. Ambos aparecen en el discurso del entrevistado $M$, en estrecha vinculación con su apelación al contra-discurso neocolonial de los recursos naturales, al atribuir a actores externos la apetencia por los recursos naturales y una cierta complicidad a los gobiernos nacionales, además de ampliar el problema a toda América Latina:

El control del territorio es uno de los problemas con los que estamos seriamente comprometidos, me parece que la extranjerización y ocupación efectiva del territorio con la explotación de bienes comunes y recursos renovables y no renovables. Ese es uno de los grandes problemas de Latinoamérica. El estado más que regulador es un disputador que está en la posición de socio activo de estos proyectos. (Entrevistado $\mathrm{M}$ )

Es significativo que, siendo el entrevistado $\mathrm{M}$ investigador del sistema público, se refiera a otras áreas de la ciencia como irrelevantes en relación con esta problemática. La vinculación entre estas dos cuestiones no es frecuente, pero resulta reveladora de la posición adoptada por este entrevistado, de tipo de intelectual - es decir, interesado por las cuestiones de interés común - más que la de experto, sólo dedicado a hablar de su tema de especialidad: "Hacer nanotecnología no nos va a dar más soberanía, porque virtual o realmente somos territorios ocupado. Miles de hectáreas 
Biotecnología agrícola y "sojización" en la Argentina: controversia pública...

Ana María Vara • Agustín Piaz • Florencia Arancibia

se vendieron a los extranjeros, los chinos quieren hectáreas para plantar soja... iy la gobernación de Río Negro lo aprobó!"

En la cita, además, aparece la cuestión tanto del creciente papel de China en la Argentina como la de la extranjerización de las tierras, en relación con un conflicto surgido sobre el interés de inversores chinos por hacer obras de infraestructura en la provincia de Río Negro para la explotación 200.000 ha de cultivos, sobre todo, soja. Lo significativo del proyecto y que suscitó mucha resistencia es que se basa en un acuerdo entre el gobierno provincial y una empresa estatal china vinculada a la producción de energía, lo que parecería indicar un interés por la soja para la producción de biocombustibles (ARANDA, 2011).

En relación con la continuación de la ampliación del marco regulatorio, puede decirse que la preocupación por la extranjerización de las tierras, que implicó una compleja discusión durante 2010 y 2011, derivó en la sanción a fines de 2011 de la Ley de Régimen de Protección al Dominio Nacional sobre la Propiedad, Posesión o Tenencia de las Tierras Rurales No 26737, o Ley de Extranjerización de Tierras, orientada a limitar la posesión de tierras por parte de actores extranjeros. Si bien la legislación sola no alcanza y es tarea frecuente de los movimientos sociales reclamar su implementación como parte de la misma dinámica de las controversias, esta cuarta línea de extensión del marco regulatorio es el indico más claro de que la continuación de la controversia puede seguir ofreciendo nuevos resultados en términos de controlar los impactos de la incorporación de biotecnología agrícola en la Argentina.

Original recebido em: 29/11/2011

Versão final recebida em: 05/03/2012

\section{Referencias}

ABLIN, E. R. y PAZ, S. Hacia la Trazabilidad en el Mercado Mundial de Soja: una nueva mirada a la ley de la oferta y la demanda. 
Buenos Aires: Cancillería Argentina, Dirección Nacional de Negociaciones Económicas y Cooperación Internacional, 2001.

ARANCIBIA, A. "Controversias científico-regulatorias y activismo: el caso de los agroquímicos para cultivos transgénicos en la Argentina”. En: TULA MOLINA, F. y VARA, A. M., Riesgo, política y alternativas tecnológicas, en prensa.

ARANDA, D. "El tóxico de los campos". Página/12, 13 de abril de 2009. URL: http:/www.pagina12.com.ar/imprimir/diario/ elpais/1-123111-2009-04-13.html, consultado 17 de febrero de 2012. . "La soja desembarca en la Patagonia". Página/12, 8 de marzo de 2009. URL: < http://wwwpagina12.com.ar/diario/ sociedad/3-163668-2011-03-08.html>, consultado 17 de febrero de 2012.

BARSKY, O. y DÁVILA, M. La rebelión del campo. Historia del conflicto agrario argentino. Buenos Aires: Editorial Sudamericana, 2008.

BARSKY, O. y GELMAN, O. Historia del Agro Argentino. Desde la conquista hasta fines del siglo XIX. Buenos Aires: Grijalbo, 2001.

BAUER, M. (ed.). Resistance to New Technology. Nuclear Power, Information Technology and Biotechnology. Cambridge: Cambridge University Press, 1995.

BEGENISIC, F. Hacia un País Sojero. Buenos Aires: Dirección de Agricultura, 2002a.

. El Quinquenio de la Soja Transgénica. Buenos Aires: Dirección de Agricultura, 2002b.

CARRASCO, A. "Efecto del glifosato en el desarrollo embrionario de Xenopus laevis (Teratogénesis y glifosato)". Laboratorio de Embriología Molecular, manuscrito, S/F.

CONNOR, S. "Argentinian scandal prompts new gene rules". New Scientist, 14 de abril, pp. 18-19, 1988. 
Biotecnología agrícola y "sojización" en la Argentina: controversia pública...

Ana María Vara • Agustín Piaz • Florencia Arancibia

CONSEJO NACIONAL DE COORDINACIÓN DE POLÍTICAS SOCIALES. Consideraciones sobre soja en la alimentación, febrero, 2003.

HESS, D. et al. "Science, technology and social movements". En: Hackett, E. J. et al. (eds.). Handbook of Science and Technology Studies, tercera edición, pp. 473-498, 2008.

JAMES, C. Global status of commercialized biotech/GM crops: 2010 - ISAA Brief No 42, Ithaca, ISAAA, 2010.

JOYCE, Ch. "US exports genetic experiments". New scientist, 20 de noviembre, p. 15, 1986.

. "La semilla que promueve el demonte". Página/12, 1 de marzo de 2012, p. 15.

LEDESMA, M. A.: "Proteccionismo: ¿ricos versus pobres?". La Nación, 4 de octubre, suplemento Campo, p. 4, 2003.

MCADAM, D., TARROW, S. y TILLY, Ch. "Comparative perspectives on contentious politics". En: LICHBACH, M. y ZUCKERMAN, A. (eds.). Comparative politics: rationality, culture, and structure: advancing theory in comparative politics. Cambridge: Cambridge University Press, 2007.

NELKIN, D. Nuclear power and its critics: the Cayuga lake controversy. Ithaca: Cornell University Press, 1971.

. Jetport: the Boston airport controversy. New Brunswick, NJ: Transaction Books, 1974.

- (ed.) Controversy: the politics of technical decisions (tercera edición). Newbury Park: Sage, 1992.

PAGANELLI, A. et al. "Glyphosate-Based Herbicides Produce Teratogenic Effects on Vertebrates by Impairing Retinoic Acid Signaling”. Chemical research in toxicology, 23 (10), pp. 15861595, 2010.

PELLEGRINO, P. Agriculture transgénique: modes de production et utilisation de la connaissance scientifique: science, etat et 
industrie dans les cultures transgéniques en Argentine. Buenos Aires. Disertación (Doctorado en Sociología). Université Sorbonne Paris IV y Facultad Latinoamericana de Ciencias Sociales-sede Argentina, 2010.

PENNA, J. A. y LEMA, D. "Adoption of herbicide tolerant soybeans in Argentina: an economic analysis". En: KALAITZANDONAKES, N. The Economic and Environmental Impacts of Agbiotech. A Global Perspective. New York: Kluwer Academic Publishers, pp. 203-221, 2003.

PEIRETTI, R. A."Direct seed cropping in Argentina: economic, agronomic, and sustainability benefits". En: SOLNBRIG, O., PAARLBERG, R. y DI CASTRI, F. Globalization and the rural environment. Cambridge: Harvard University David Rockefeller Center for Latin American Studies, pp. 178-200, 2001.

POVERENE, M. y Cantamutto, M. "Impacto ambiental de los cultivos trangénicos”. Ciencia Hoy, v. 13, n. 75, junio-julio, pp. 26-37, 2003.

RAPOPORT, M. Historia económica, política y social de la Argentina (1880-2000). Buenos Aires: Ediciones Macchi, 2000.

RECA, L. G. y PARRELLADA, G. H. El Sector Agropecuario Argentino: aspectos de su evolución, razones de su crecimiento reciente y posibilidades futuras. Buenos Aires: Editorial Facultad de Agronomía, 2001.

RUSE, M. y CASTLE, D. (eds). Genetically Modified Foods. Debating Biotechnology. Amherst, NY: Prometheus Books, 2002.

TARROW, S. "Cycles of collective action: Between moments of madness and the repertoire of contention". Social Science History, v. 17, n. 2 (verano), pp. 281-307, 1993.

TRIGO, E. J. Quince años de cultivos genéticamente modificados en la Argentina. Buenos Aires: Argenbio, 2011.

TRIGO, E., CAP, E. "The impact of introduction of transgenic crops in Argentinean agriculture”, AgBioForum, 6(3) pp. 87-94, 2003. 
Biotecnología agrícola y "sojización" en la Argentina: controversia pública...

Ana María Vara • Agustín Piaz • Florencia Arancibia

TRIGO, E. J. et al. Los transgénicos en la agricultura argentina: una historia con final abierto. Buenos Aires: Libros del Zorzal-IICA, 2002.

VAN ZWANENBERG, P. "La regulación de la biotecnología y las políticas de regulación tecnológica”. En: TULA MOLINA, F. y VARA, A. M. Riesgo, política y alternativas tecnológicas, en prensa.

VARA, A. M. "Transgénicos en Argentina. Más allá del boom de la soja". Revista Iberoamericana de Ciencia, Tecnología y Sociedad, Vol I, N 3, septiembre, pp. 101-129, 2004.

. "Argentina, GM nation. Chances and choices in uncertain times", country case, NYU Project on International GMO Regulatory Conflicts, 2005. URL: <http://www.law.nyu.edu, consultado 15 de mayo de $2011>$.

. Anti-imperialismo y literatura: la emergencia del contradiscurso neocolonial de los recursos naturales en América Latina. Riverside. Disertación (Doctorado en Estudios Hispánicos). University of California, Riverside, 2009.

. "Protesta ambiental latinoamericana". Voces en el Fénix n. 2, pp. 36-41, 2010. < http://www.vocesenelfenix.com/content/ protesta-ambiental-latinoamericana $>$.

. "Riesgo, recursos naturales y discursos. El debate en torno a las tecnologías y el ambiente en América Latina”, aceptado para publicación en la Revista Tecnología y Sociedad, en prensa.

\section{Listado de entrevistados:}

-Entrevistado A: miembro de una asociación argentina vinculada a la industria biotecnológica. Realizada en Buenos Aires, 18 de febrero de 2011.

-Entrevistado B: director de una empresa argentina dedicada a la consultoría sobre negocios agrícolas, autor de numerosos estudios sobre el impacto de la introducción de transgénicos en la Argentina. Realizada en Buenos Aires, 15 de abril de 2011. 
-Entrevistado C: director de asuntos regulatorios para Argentina de empresa transnacional de semillas y agroquímicos. Respuestas enviadas por email el 5 de julio de 2011.

-Entrevistado D: miembro de CONABIA y profesora de la Facultad de Agronomía de la Universidad de Buenos Aires (UBA). Realizada en Buenos Aires, 16 de marzo de 2011.

-Entrevistado E: experto en genética y biotecnología del Instituto Nacional de Tecnología Agropecuaria (INTA). Realizada en Buenos Aires, 28 de marzo de 2011.

-Entrevistado F: experto en genética y biotecnología de la Facultad de Ciencias Exactas y Naturales de la UBA; del Consejo Nacional de Investigaciones Científicas y Técnicas (CONICET); y de un centro de investigación financiado por empresas y asociaciones que apoyan la biotecnología agrícola. Realizada en Buenos Aires, 18 de abril de 2011.

-Entrevistado G: coordinador de un programa vinculado a la problemática de la deforestación en la sede argentina de una ONG ambientalista internacional. Realizada en Buenos Aires, 4 de marzo de 2011.

-Entrevistado $\mathrm{H}$ : fundador de una organización de movimiento social argentina dedicada a cuestiones rurales. Realizada en Buenos Aires, 17 de marzo de 2011.

-Entrevistado I: coordinador de un programa dedicado a clima y deforestación en la sede argentina de una ONG ambientalista internacional. Realizada en Buenos Aires, 4 de mayo de 2011.

-Entrevistado J: médico y miembro de una organización de movimiento social argentina dedicada a cuestiones rurales. Realizada en Buenos Aires, 6 de mayo de 2011.

-Entrevistado K: diputado nacional por la provincia de Córdoba por un partido de centro-izquierda. Realizada en Buenos Aires, 21 de marzo de 2011. 
Biotecnología agrícola y "sojización" en la Argentina: controversia pública...

Ana María Vara • Agustín Piaz • Florencia Arancibia

-Entrevistado L: funcionario de la Cancillería Argentina. Realizada en Buenos Aires, 29 de marzo de 2011.

-Entrevistado M: investigador de la Facultad de Medicina de la Universidad del Buenos Aires y del Consejo Nacional de Investigaciones Científicas y Técnicas (CONICET). Realizada en Buenos Aires, 13 de abril de 2011.

\begin{abstract}
Agricultural biotechnology and "soybeanization" in Argentina: public controversy, construction of consensus, and expansion of the regulatory framework

The introduction of genetically modified (GM) crops in Argentina within a for-export framework since the mid-1990s was led by herbicide-tolerant soybean. In the beginning, public resistance to this introduction was not strong enough to have an impact on the planning of promoters; among those, the establishment of a regulatory framework that focused exclusively on biosafety. However, we have found that new, complementary norms have been established lately. Those may be considered an informal expansion of the regulatory framework, respond to local critisim, and pay attention to local issues related to environmental, social and health impacts. Previously, two more outcomes were reached: the setting up of a public debate on the introduction of transgenic crops; and a consensus, shared by both pro- and anti-GM actors, regarding certain negative social and environmental impacts of this introduction.
\end{abstract}

Keywords: genetically modified crops, herbicide-tolerant soybean, resistance to technology, regulatory framework, Argentina. 